\title{
A Case Study On Auto Socialization In ONLINE Platforms
}

\author{
Lars Aagaard-Kirkeby
}

MSc. In It, learning and organizational change. Aalborg University CPH. Denmark. Ph.D.-stud. in spe. DTU (Technical University of Denmark) Lars Aagaard-Kirkeby.

\begin{abstract}
Auto socialization Theory[1][2]is a socialization theory, introduced by Swedish Social Psychology Professor Emeritus Lars Dencik, Roskilde University Center (RUC) on how humans socialize, attempting to be a part of a group by using a taxonomy in three stages. Even though the theory is based on socialization amongst minors, the adult human uses Auto socialization to navigate everyday life in all its aspects of interaction, including communications, collaborations through online platforms. By focusing on the Auto socialization aspect in an online context, it is possible to explain why online platforms e.g. Facebook, World of Warcraft are so successful in maintaining and increasing the numbers of stabile audience and why other platforms, offering learning on a massive scale, (MOOC) facing dropout rates in the high $80-90$ 's[3][4][5].
\end{abstract}

\section{KEYWORDS}

Auto socialization, MOOC's, online platforms, online education, e-living, e-learning.

\section{INTRODUCTION}

Approx. 1 year ago in preparation of a master's thesis, contact was made to The Emeritus Professor Lars Dencik. Asking him on issues of socialization behaviors in a physical world vs. socialization in a digital environment, the modus was established by the professor, as the same way to socialize. Hence making the auto socialization process available in an online context.

The Auto socialization theory[1]is based on observations of infant children in their attempts to become part of groups. Lars Dencik came to the conclusion, that children were an active contributor to their own socialization. Until that time, the common understanding of socialization was based on, children needed to be socialized by others, not playing an active part in this development. The theory is as mentioned, based on how the individual is becoming a part of a group due to contemplation and the imitation of the significant other in a group. If imitated, the individual will conform with the groups values and thereby becoming a part of the group. The theory suggests a taxonomy as follows: Contemplation - Imitation - Conformation. In an online context, the center of the theory, the imitation process is conducted through a representation of the significant other. This representation being expressed in pictures, wall notifications, texts in form of greetings, updates, instructions, live feeds e.g. By offering the onboarding user a basis of imitation, the user will be able to assess in accordance with the auto socialization taxonomy, weather to continue the socialization attempt or to abort the attempt and restart the taxonomy.

\section{Presentation Of Hypothesis}

In the context of Professor Denciks clarified socialization thesis, a new hypothesis was made to counter the field study on human's possible socialization in online platforms. 
"Human individuals socialize in the digital world using a taxonomy called "auto socialization". The taxonomy consists of three parts: contemplation, imitation and conformation. If the human individual cannot imitate or reflect in the significant other or the representation of the significant other, the individual will abort the attempt to socialize and not be a part of the digital/ physical group."

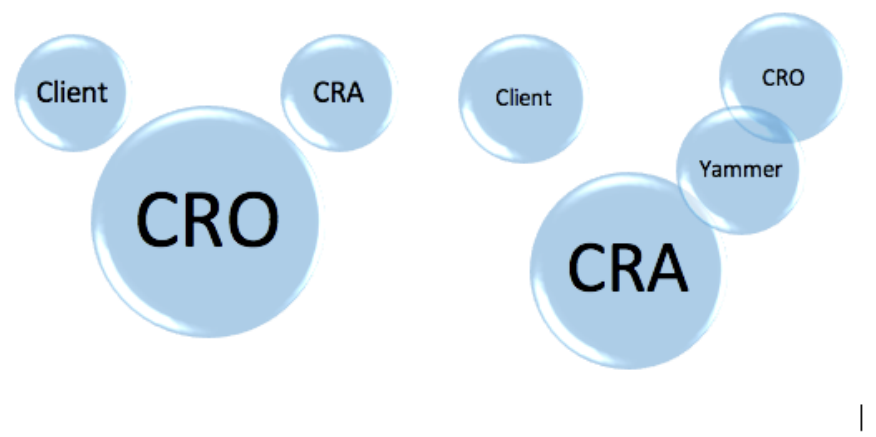

Figure 1. Yammer sociotop in the field study.

\section{FIELD STUDIES}

By implementing an online communication and collaboration platform (Yammer) in the CRO's (Clinical Research Organization) communications strategy, the company intended to create an online forum. In this forum, they intend to connect both on a social level as well as a professional level with the outplaced Clinical research associate (CRA).

Further more and more importantly for the case study, the implementation would provide data on the socialization attempt according to Lars Denciks Theory on Auto Socialization in this case study.

\subsection{Purpose}

In the context of the hypothesis, the Yammer implementation was mend to create an understanding of the taxonomy. Especially what the significant other meant to the taxonomy and how the representation of the significant was being conducted in an online environment, was important to acquire knowledge of.

Furthermore, the Yammer implementation would show if, the outplaced associates would imitate the significant other and become a part of the clinical research organization company or reject the imitation process and reject the socialization attempt entirely.

\subsection{METHOD}

In an empirical critical case study lasting 12 weeks on implementing the Yammer platform in a Nordic Clinical Research Organization (CRO), a group of 5 outplaced associates and senior management in Danish field office participated in interviews before and after implementation of the Yammer platform. 

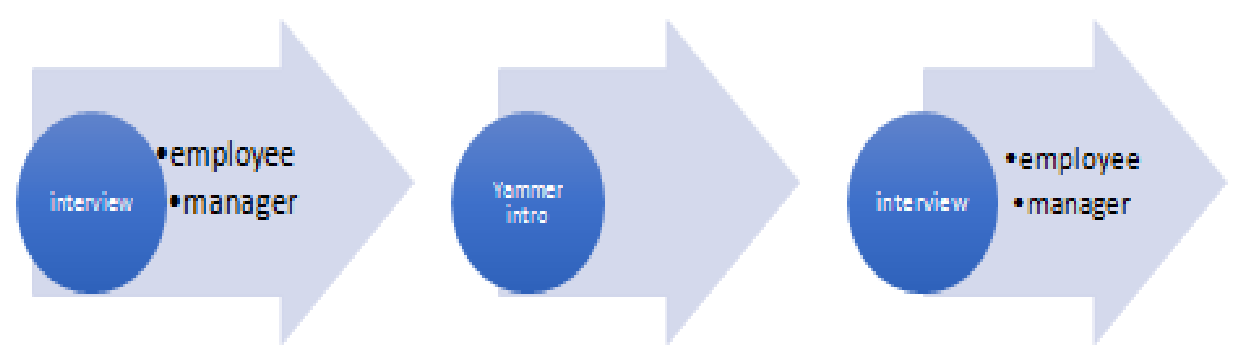

Figure 2. Field study progression through 12 weeks.

By using qualitative interviews before and after the intervention, Thematic Analyzing [6][7] was used to secure themes of the process. In order to truly identify the significant others values by addressing the themes, Scheins organizational culture analysis [8] was applied to reveal the significant others values or imitation basis for the outplaced CRA to imitate in the taxonomy.

Central to the method was the fact, that the Outplaced Clinical Research Associates (CRA) didn't have a daily consistent contact to their employer at the field office (CRO). Senior management from CRO was in-house personnel from the Danish field office participating as significant others in the Auto socialization taxonomy.

\subsection{RESULTS}

The case study shows, that the outplaced associates all have individual reasons, grounded in their everyday life and social circles, not to imitate the significant other at the CRO in the online platform environment and therefore the socialization attempt by introducing Yammer to the company was rejected. Furthermore, the organizational culture analysis of the significant others imitation basis, reveals that the outplaced associates, did not share the organization core value as the staff of the Danish country office, thereby not imitating or reflecting the significant others values.

Other results show a conflicted and divided management as a result of the implementation process, not being able to create a sufficient significant other imitation basis, for the outplaced personnel to imitate or reflect in.

\section{DISCUSSION}

Initially I want to quote Professor Lars Dencik on the subject on "seeing the overlooked". Through this last year working on this hypothesis, it became clear to me, how hard it is to convince others, and even myself to some extent, in the ongoing research:

"The revolutionary task of science is.... through the contrasts to notice phenomenon's previous ignored existence and integrating them in a new - but always provisionally insight. And through this, realize or construct a new object to investigate further" [9, p. 61]. 


\subsection{Why Auto Socialization?}

From a previous made literature review regarding MOOC's and the dropout rates inspired by Professor Emeritus Alan Taite Open University UK, it was noticed how massive and generally occurring these rates were. Dropout percentages in its 80-90's, occurred [5] [4] [3]throughout various cultural differences, through various learning cultures, and through multifarious academic disciplines. The dropout rates didn't pay attention to cultures, learning cultures, traditions. The only common denominator seemed to be the human socialization process and how it's intertwined in human lives, how humans conduct their lives and how they relate to their conspecifics.

\subsection{Is SocialiZation An URge?}

This question could be answered poetically by priest and poet from the 1700 century John Donne and his poem "No man is an island entire of itself". He recognized the meaning of others in his life and his in others.

All human being needs another entity in its life in the beginning. The first basic communication from the child to the caretaker are mimicking facial expressions and the baby's body movement in accordance with the caretaker's voice [10].

If the baby's communications attempts are not recognized or ignored by the caretaker, the infant might slip into a passive state, subsequently inflicting lifelong trauma of affiliation and trust issues.[11]. In [12] the individual is locked in a companionship with the generalized other, forming and reforming identities, responding to the others reaction to newest edition of identity tryouts.

Auto socializing theory is "built" into children's social agent in relation to be a part of a group seeking out others. In a child's development (Bruner, 1990) (Dencik L., 2006), they are virtually encoded to seek each other via the connection to the significant other in order to beginning creation of identities and being a part of a group of children playing. Their socialization may seem banal in an adult perspective where socialization is not based solely on the urge / need or in an identity development perspective [13].The individual's own experiences of previous socialization attempts and also desire for alliances, protection, information or simply company may influence the socialization attempts. If an urge to socialize in the adult context, can be interpreted different, maybe words like "duty", "coercion" or obligation" or "desire" can be used to describe the inner or outer influence to participate in every day work and also in online communications and collaborations.

\subsection{BY THE NUMBERS}

Figures from 2015[14] clearly states that social and e-learning platform on a global scale is on the rise. Thus $80 \%$ of fortune 500 companies were using social/collaborative platforms( Yammer, Chatter, Social Cast) in 2012[15], 63\% of all Fortune 500 companies stated, social business would be imperative to their business within 3 years. An increase of expectations of more than $250 \%[16]$.

At the same time more than 35 mio. Users have been sign up for a MOOC from more than 500 Universities and more than 4200 courses over all. In 2015 Coursera gained more than 7 mio. New participants [14]. The trend is to introducing more online learning courses and new platforms. 


\subsection{Human/TEChNOLOGY EvOlution}

The technological evolution is so much faster than the human evolution. The overwhelming construction of new and better platforms are almost daily events. Each featuring new ingenious ways to connect and collaborate or sharing knowledge.

But no matter how smart or fast these platform applications might appear to be, they have no value in an learning/auto socialization context, if the application fails to connect to the user. Just because they are there on the screen, doesn't make the students/employees/ individuals pay attention or using it.

\section{The Learning Triangle And The Social Aspects Of Mastery LEARNING}

In accordance with Knud Illeris' general learning theory[17], all learning consists of 3 dimensions and 2 processes. This learning philosophy is common to northern European countries and public schools in general throughout this area.

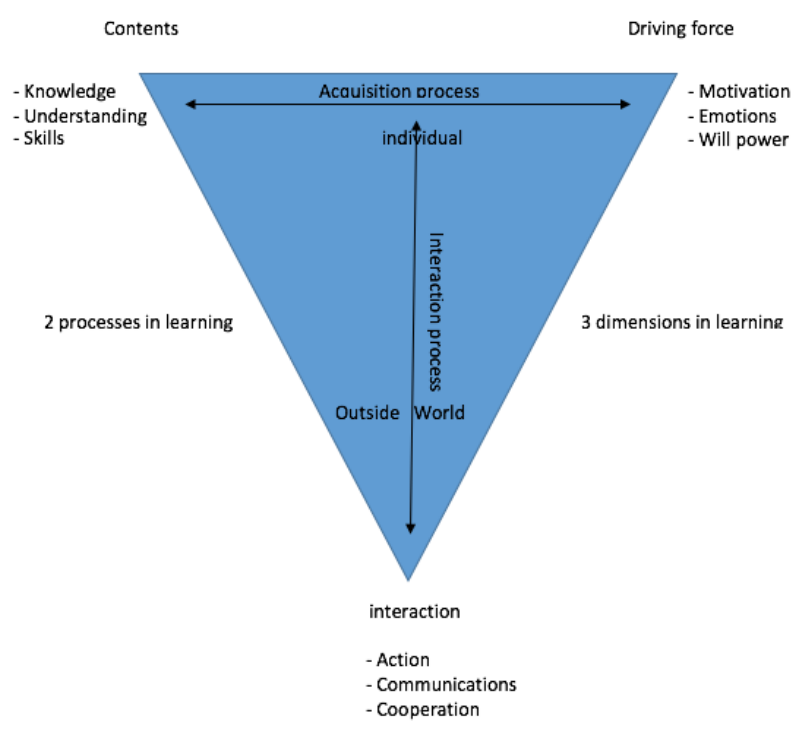

Figure 3. Illeris learning triangle translated. Illeris 2000.

One of the displayed dimensions is the "interaction dimension". This dimension is about the interaction between the individual and the outside world, development of social skills as learning occurs. Bigger commitment to the interaction will give greater learning possibilities. [18]

If the interaction dimension is missing entirely from the fictive learning situation as illustrated, the learning situation will be reduced to a passive memorization technique on repetition (rote learning). The subject will stay in the acquisition process, not verbalizing or interact what is taught. This kind of learning is also called "Gas station attendant pedagogy". The visualization is a teacher filling up passive pupils head with information, like filling gasoline on a car.

\subsection{MASTERY LEARNing}

In a MOOC context, ex. Coursera, based their online learning on "Mastery learning" by Carrol \& Bloom. The mastery learning will guide the user through an assignment through questions and 
finally do a test. If the user fails the test, mastery learning technique dictates repetition until the level is passed or mastered

This method is appropriate to use in online learning environment for several reasons:

1. The mastery Learning method focuses on the individual, measuring progress throughout the levels presented to the individual.

2. It produces data on the user and data to the provider on courses activity. Also data in regards to overall program dynamics will be available to the providers.

3. The learning method gives the provider documentation that transfer of information or training has occurred.

4. The learning method affixes the user in a inactive position and not in a active correlation with the surroundings.

The method doesn't provide the interaction dimension and in accordance with the general learning theory by Illeris, learning will not take place. From a perspective covering the auto socialization, users of the featured online learning platform subjects to this method, are likely to failing in maintaining basic social relations in accordance the auto socialization theory, hence the vast percentage in dropout rates.

\subsection{MASTERy Learning ONLINe ANd In A CoOperate World}

The mastery learning method is widespread throughout online e-learning providers and in the private sector in internal courses and web seminars in businesses all over the world. The traditional way of conducting these in companies, are by slideshows/PowerPoints and a commentators voice following the slideshows. The occasional test at the end of every topic, must in some cases, be past the $80 \%$ mark of correct answers, or the topic will be repeated. Sometimes the participants will have 3 tries for success, in other sessions, one test with less than $80 \%$ correct answers, will make you fail the topic.

In relation to the conducted case study, it has been observed employees circumvent highly expensive, complex and long lasting onboarding programs by using a smartphone. They were taking pictures of essential information for the hourly test, on the hour, every hour, 8 hours a day for 3 days.

This goes to show, even though humans tends to evolve slowly, we are second to none at using tools even if we don't pay attention. Not being an active part of a onboarding program or web seminar, we can deliberately circumvent any web based or online event in a company, because we don't connect the right way.And because it is a web based seminar, nobody notice this. The learning department in the company will be able to document some sort of transferring of information to the users. And the users will have a documented seminar, course or a mandatory onboarding program under the belt.

There will probably be learning specialists reading this, who will cry out: "Not in my company..." and the answer is... "Yes.. also in Your company". As the auto socialization theory, according to Professor Lars Dencik is a general socializations theory, it also apply for you. People seems to "disconnect" when the socialization attempt is disrupted, but the inner or outer influences keeps the participant in the loop so to speak, taking action to preserve documentation of interaction, but not really participating. 
The danger of a curriculum guided events in a mastery learning matrix, may be the appreciation of percentages in test scores. Because the mastery learning method is based on individual test results.

Constructing any learning technology in accordance with known human skills, such as social skills maybe a far better way to spend vast amounts of resources, while including new employees or educating employees, not excluding them in a technical race of the developers.

It would surely be interesting to examine weather this reaction is a natural reaction and if there is a causality to a disconnected socialization attempt during learning events.

And it would be even more interesting to examine if the opposite reaction to mastery learning and the finishing rates (approx. 7-9 \%) in the MOOCs also have causality, due to the lack of being exposed socially.

\section{THE NeCESSARY SOlution Of ONLINE LeaRning}

Professor Emeritus Alan Tait Open University UK, held an online lecture spring 2015 to Aalborg University in which he stated, that in order to fulfil educational demands on a global scale, online learning would have to be seriously considered. As online learning was the only platform or media, place even, who would be able to contain such vast numbers of students.

But should Online Platforms in its current form even be considered, in regards to the massive dropout rates? Is it sufficient to state, that a large number of starting student, justifies massive dropout rates. As long a sufficient number of student pass the course?

Numbers are hard to ignore. [5] refer to a University of California Berkley MOOC with 50.000 signed up participants and a dropout rate at $93 \%$. Only 3500 finished the course.

In its current state, leaders in online learning platforms (MOOC's) are starting to charge small fees, from my point of view, in order to make courses matter, or from the notion :" if it cost me, it must be important enough to finish". Of cause it's also to make money, but the thought is there. Two years ago you would only have to pay, if you wanted a certificate" to go with the course". Even if it is a cheap shot, making a reference to fast food, there are some truth and similarities serving the same, the same way, all over the world. The "McDonaldization"[4] of online learning is hard to miss.

\section{E- Learning Paradigms - Where do We Go From Here?}

In this article the focus has been on the discrepancy between the current state on e-learning in the online environment and the human condition in this environment. Problem is, the one half of the discrepancy, the current state of online learning, has no current state, but continues to evolve, making the current state, a state of change. Whereas the other part of the discrepancy, the human condition, haven't changed significantly since before the building of the first pyramids on the African continent.

Rather than asking how to change or diminish the discrepancy between humans and learning technology, the why question is so much more relevant in this discourse. A re-launch of online learning technology development focusing on human characteristics, may yet be the biggest progress in the human phylogeny since the arrival of the mobile telephone in the 1980's. 
There is a lot to be gained in this re-launch. Time, money, resources, dynamics in learning and seminars, even environmental impacts. Companies would be able to reconstruct its learning capabilities in all its aspects, thereby reducing costs on physical sessions in all its aspects. All this while gaining access to employees accumulated knowledge pool, using their strengths to solve organizational and strategic issues.

Also in a commercial online learning context there would be significant benefits in solving the drop out issues. The platform media would gain thoroughness and a dawning conviction of a sustainable and viable product, over time creating a convincing audience and a sound online elearning tradition.

If we are to overcome the constrictions of the physical presence in online learning, we have to turn away from the present paradigm of focusing on bandwidth, server surplus and repetitions without participation. If employees, students or just humans are to receive training, learning, collaboration, it is vital to focus on the human condition, instead of charging up the digital battlefield of inventions. Because that battle is already lost. The dropout rates is there to prove it.

In the search of inspiration, we could cast a glimpse at the neighboring areas e.g. the gaming world. How do they solve the problem on retaining users? E-games (Counter strike teams) growing rapidly, World Of Warcraft clans living on for years.

\section{Perspective}

The digitalization is making its mark on everyday life. In our corner of the world, computers and ICT (information/communication technology) influences our lives in ways we are not even conscious about.

This article focus on the individual use of online based platforms, social media and learning is conditional to the pace, which the technology develops right now. "E-learning" and "Social media" are not only everyday buzzwords, but an expectation to the individual in regards to learning interaction, sharing and collaboration regardless of life situations (pupils, students, adults and senior citizens) This interaction with online platforms represent a presence from public school, over semi social platform for collaboration, to a senior citizen's life with a link to public health centers. There are many more representatives of online connections besides the ones mentioned here and the above was only mentioned as an example.

As "Interaction with e-learning" or "interactions on social media" is not sufficient in the description of a citizen's entire life in relation to the cohesion with the digital world, an introduction of the term E-living is appropriate. This consideration is not an expression of the individuals narrow digital interaction in a work/spare time context, but an expression of an entire life in a digitalized society.

\section{Conclusions}

The basis of this paper, the case study on auto socialization in an online platform shows, individuals will not continue the socialization attempt if there is no basis for imitating in the significant other.

This point to a focus on the individual's active participation in their own socialization, and the discontinuance of the socialization attempt is a natural reaction to a process not involving its participants, thus the focus on the reinvention of the e-learning paradigm based on human acquired or inherent skills and not on inventions of technology. 
Per the Auto socialization taxonomy, the focus must be on, creating a strong and inclusive representation of the significant other, to allow the participants, to continue in their individual socializations attempts with the online media.

Finally, in continuation of the Dencik quote" to notice the ignored phenomenon", the phenomenon here is the generally occurring dropout rates or as seen in the case study, associates not willing to socialize. The rates are not only the results of insufficient methods or insufficient imitation basis, but a natural occurring phenomenon, when the individual discontinues its socialization attempts.

\section{ACKNOWLEDGEMENT}

Author would like to acknowledge Emeritus Professor Lars Dencik, Roskilde University Center DK (RUC) for his contribution to the thesis and the subsequently article and his invaluable input in regards to the "auto socialization" theory in the article. The author also want to acknowledge Emeritus Professor Alan Taite, Open University London UK, for his contribution through lectures on the MOOC perspective.

\section{REFERENCES}

[1] P. S. Jorgensen and L. e. A. Dencik, children and family in postmodern society, 5th edition ed., P. p. Lars Dencik, Red., Copenhagen: Hans Reitzel A / S, 1999, p. 540th

[2] L. Dencik, "About to-view, go-sight and error-sight.," The man in the post-modernization. 1st edition 2nd edition ed., Billes $\varnothing$ \& Baltzer, 2006, pp. 34-75.

[3] R. F. P. C. S. E. Kizilcec, "Deconstructing Disengagement: Analyzing Learner Subpopulations in Massive Open Online Courses, 'LAK 13, 2013.

[4] Kjærgaard et al, "MOOCs - Prospects for UC-sector in Denmark," the journal Learning and Media (LOM), Volume 6 No. 112013.

[5] T. R. P. P. a. W. S. Liyanagunawardena, 'Dropout: Mooc participants'perspective.,' In Dropout: Mooc participants' perspective., Lausanne, 2014.

[6] M. Bjerrum, 1st edition ed., Copenhagen K, Denmark: Academic Publishing, 2005.

[7] V. Braun and V. Clarke, "Using thematic analysis in psychology," Qualitative Research in Psychology, pp. 77-101, March 2006.

[8] E. H. Schein, 2nd edition ed., Publisher Valmuen, 1994, p. 378th.

[9] L. Dencik, Man in postmoderniseringen- of childhood, family and identities in flux, 1st edition 2nd edition ed., Billes $\varnothing$ \& Baltzer, 2006.

[10] D. N. Stern, the first 6 months, 2nd edition 3rd edition ed., Routledge, 1979.

[11] E. H. Erikson, 1st edition ed., W. W. Norton \& Company, 1997.

[12] G. H. B. (. Mead, 1st edition ed., Baldwin, J. D., Ed., Sage Publications, 1986, p. 168th.

[13] J. Mezirow, A guide to Transformative and Emancipatory learning, Jossey-Bass Inc., 1990.

[14] D. Shah, 'By the numbers, "19 July 2016. [Online]. 
International Journal of Managing Information Technology (IJMIT) Vol.9, No.1, February 2017

Available: https://www.classcentral.com/report/moocs-2015-stats/. [By retrieved or displayed 11 August 2016].

[15] J. S. Brady, "The corporate social network as an internal communication medium," UMI Dissertation Publishing, 2012.

[16] Chiron, palms, Phillips and Kruschwitz, MIT Management Review, North Hollywood, 2012.

[17] K. Illeris, Learning, 3rd Edition ed., community literature, 2015, p. 300 TEC.

[18] E. A. Knud Illeris (ed.), learning theories six current understandings, 1st edition 3 edition ed., Roskilde University Press, 2007, p. $160^{\text {th }}$

\section{AUTHOR}

Lars Aagaard-Kirkeby is a Ph.D.-stud. in spe. DTU (Technical University of Denmark)2014-2016 MSc. In It, learning and organizational change. Aalborg University CPH. Denmark.2000-2004 BA in social Science.

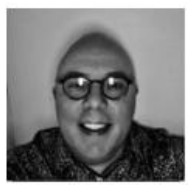

